\title{
AN EXTENSION OF HORN'S THEOREM FOR A SPACE OF LOCALLY INTEGRABLE FUNCTIONS
}

\author{
HERNAN R. HENRIQUEZ \\ EDUARDO A. HERNANDEZ \\ Universidad de Santiago de Chile \\ Departamento de Matemática \\ Casilla 5659, Correo 2, Santiago \\ SANTIAGO - CHILE
}

(Received April 24, 1991 and in revised form July 16, 1991)

ABSTRACT. In this paper we extend the fixed point theorem of Horn's to the space of locally integrable functions from $(-\infty, 0$ linto a Banach space $X$.

KEY WORD AND PHRASES. Locally integrable functions, fixed point theorem, Banach spaces.

1991 AMS SUBJECT CLASSIFICATION CODES. Primary 47H10.Secondary 34K20.

1. INTRODUCTION.

Horn's fixed point theorem [ 3 ] has numerous interesting applications, espe cially to obtain periodic solutions of differential and retarded functional differen tial equations. In this subject we refer to the paper of Burton and Dwiggins [ 1 ] as well as those referencesmentioned in it. In these applications, the problem of existence of periodic solutions is reduced to the existence of a fixed point in the phase space associated with the equation. Consequently, in order to apply Horn's theorem it is necessary that the phase space be a Banach space. Nevertheless, this condition is not appropriated for retarded functional differential equations with infinite delay and, in general, for abstract differential equations. For these reasons Burton and Dwiggins estab1ished an extension of Horn's theorem to the Fréchet space $C\left((-\infty, 0] ; \mathbb{I R}^{n}\right)$ endowed with the compact -open topology. This extension permits consideration of equations whose initial conditions are continuous functions. However, in many initial values problems the space of continuous functions is not the most appropriate, being necessary to consider integrable functions. This ocrurs, for example, in some models used in control theory ( [ 2 ] ).

We now state Horn's theorem [ 3 ].

THEOREM 1. Let $\mathrm{X}$ be a Banach space, $\mathrm{S}_{\mathrm{o}} \subseteq \mathrm{S}_{1} \subseteq \mathrm{S}_{2}$ convex subsets of $\mathrm{X}$ with $\mathrm{S}_{0}$ and $\mathrm{S}_{2}$ compact and $\mathrm{S}_{1}$ open in $\mathrm{S}_{2}$. Let $\mathrm{P}: \mathrm{S}_{2} \longrightarrow \mathrm{X}$ be a continuous map. Suppose that for some integer $m>0$ the following conditions hold :

a) $\mathrm{P}^{\mathrm{j}}\left(\mathrm{s}_{1}\right) \subseteq \mathrm{s}_{2}$, for $1 \leqslant \mathrm{j} \leqslant \mathrm{m}-1$, and

b) $\mathrm{P}^{\mathrm{j}}\left(\mathrm{S}_{1}\right) \subseteq \mathrm{S}_{\mathrm{o}}$, for $\mathrm{m} \leqslant \mathrm{j} \leqslant 2 \mathrm{~m}-1$.

Then $\mathrm{P}$ has a fixed point in $\mathrm{S}_{0}$.

In this note we use the line of reasoning of Burton and Dwiggins [ 1 ] to 
extend the Horn's theorem to a Fréchet space of locally integrable functions.

In the sequel we will denote by $X$ a Banach space with norm $\|\cdot\|$. We will designate by $B$ the Frêchet space consisting of the equivalence classes of functions $\varphi:(-\infty, 0] \longrightarrow X$ which are locally integrable in the Bochner sense, endo wed with the topology $\tau$ induced by the family of seminorms

$$
p_{n}(\varphi)=\|\varphi(0)\|+\int_{-n}^{0}\|\varphi(\theta)\| d \theta, \quad n \in \mathbb{N}
$$

We will represent by $G$ the set formed by all continuous and increasing func tions $g:(-\infty, 0] \longrightarrow[0,1]$ such that $g(0)=1$ and $g(\theta) \longrightarrow 0$, as $\theta \longrightarrow-\infty$. For each $g \in G$ we will write $B_{g}$ to denote the space of the equivalence classes of locally integrable functions $\varphi:(-\infty, 0] \longrightarrow X$ such that

$$
\|\varphi\|_{g}=\|\varphi(0)\|+\int_{-\infty}^{0} g(\theta)\|\varphi(\theta)\| \mathrm{d} \theta<\infty .
$$

It is easy to see that $\left(B_{g},\|\cdot\|_{g}\right)$ is a Banach space.

The aim of this paper is to prove that theorem 1 holds if we susbtitute the arbitrary Banach space $X$ by the Fréchet space $(B, \tau)$.

2. RESULTS.

In this section we will prove that Horn's theorem is also true in the space $(B, \tau)$.

The proof is a consequence of the following four lemmas, that relate the Banach spaces $B_{g}$, for $g \in G$, with the space $(B, \tau)$.

LEMMA 1. Let $g \in G$. The following conditions hold :

i) The inclusion mapping $i: B_{g} \longrightarrow B$ is continuous.

ii) If $\mathrm{S}$ is a bounded subset of $B_{g}$ then the inclusion map $i:(\mathrm{S}, \mathrm{r}) \longrightarrow \mathrm{B}_{\mathrm{g} \cdot \mathrm{h}}$ is continuous for every $h \in G$.

Proof. Since $B$ is a Fréchet space whose topology is generated by the seminorms $p_{n}, n \in \mathbb{N}$, to prove assertion $\left.i\right)$ it is sufficient to observe that

$$
\begin{aligned}
g(-n) p_{n}(\varphi) & =g(-n)\|\varphi(0)\|+g(-n) \int_{-n}^{0}\|\varphi(\theta)\| d \theta \\
& <\|\varphi(0)\|+\int_{-n}^{0} g(\theta)\|\varphi(\theta)\| d \theta \\
& <\|\varphi\|_{g},
\end{aligned}
$$

which yields that $p_{n}(\varphi)<\|\varphi\|_{g} / g(-n)$, for every $\varphi \in B$.

Next we will prove $i i)$. If $S$ is a bounded subset of $B_{g}$ then $S \subseteq B_{g h}$, for each $h \in G$. Let $M>0$ be a constant such that \|\|$_{\mathrm{g}} \leqslant M$ for all $\varphi \in S$. For each $\varepsilon>0$ there exists a positive integer $n$ such that $\|h(\theta)\|<\frac{\varepsilon}{4 M}$, for every $\theta<-n$. 
Let $\varphi, \psi \in \mathrm{S}$ be such that $\mathrm{p}_{\mathrm{n}}(\varphi, \psi) \leqslant \frac{\varepsilon}{2}$. From the definition of $\|\cdot\|_{\mathrm{gh}}$ we obtain the following estimate

$$
\begin{aligned}
& \|\varphi-\psi\|_{g h}=\|\varphi(0)-\psi(0)\|+\int_{-\infty}^{0} g(\theta) h(\theta)\|\varphi(\theta)-\psi(\theta)\| d \theta \\
& \leqslant\|\varphi(0)-\psi(0)\|+\int_{-\mathrm{n}}^{0} g(\theta) h(\theta)\|\varphi(\theta)-\psi(\theta)\| \mathrm{d} \theta \\
& +h(-n) \int_{-\infty}^{-n} g(\theta)(\|\varphi(\theta)\|+\|\psi(\theta)\|) d \theta \\
& \leqslant\|\varphi(0)-\psi(0)\|+\int_{-n}^{o}\|\varphi(\theta)-\psi(\theta)\| d \theta+2 M h(-n) \\
& =p_{n}(\varphi-\psi)+2 M h(-n) \\
& \leqslant \varepsilon \text {, }
\end{aligned}
$$

which shows that the inclusion $S \longrightarrow B_{g h}$ is continuous for the relative topology in $\mathrm{S}$.

Concerning this result, it is worth mentioning that, in general, the inclusion $i:(S, \tau) \longrightarrow B_{g}$ is not continuous. In fact, let us consider the space $X=C$ and let $g$ be the function defined by $g(\theta)=|\theta|^{-2}$ for $-\infty<\theta<-1$ and $g(\theta)=1$ for $-1 \leqslant \theta \leqslant 0$. From this definition it follows that $g \in G$. Let us define, for each natural $n$, the function $\varphi_{n} \in B$, which equals $g$ in $(-\infty,-2 n] \cup[-n, 0]$ and $\varphi_{n}(\theta)=g(\theta)+|\theta|$, for $\theta \in(-2 n,-n)$. It is easy to see that these functions belong to $B$ and that $\varphi_{n} \longrightarrow g$, as $n \longrightarrow \infty$, in the topology of $B$. We can also see that the subset $S=\{g\} \cup\left\{\varphi_{n}: n \in \mathbb{N}\right\}$ is bounded in $B_{g}$. However,

$$
\left\|\varphi_{n}-g\right\|_{g}=\int_{-2 n}^{-n}|\theta|^{-1} d \theta=\ln 2,
$$

which shows that the sequence $\left(\varphi_{n}\right)_{n}$ does not converge to $g$ in $B_{g}$. Hence the inclusion $S \longrightarrow B_{g}$ is not continuous.

LEMMA 2. Let $S$ be a compact subset of $(B, \tau)$. Then for every pair of constants $\mathrm{a}, \mathrm{b}>0$ and every positive integer $\mathrm{k}$ there exists an increasing and continuous function $q:[-k,-k+1] \longrightarrow[0, \infty)$ such that $q(-k+1)=a$ and

$$
\int_{-k}^{-k+1} q(\theta)\|\varphi(\theta)\| d \theta \leqslant b,
$$

for every $\varphi \in \mathrm{S}$.

Proof. For each $\varphi \in B$ we will denote by $R(\varphi)$ the restriction of $\varphi$ to the interval $[-k,-k+1]$. It is clear that the mapping $R: B \longrightarrow L^{1}([-k,-k+1] ; X)$ is continuous, which implies that the set $R(S)$ is compact in the space $L^{1}([-k,-k+1] ; X)$. Consequently, there exist functions $\varphi_{1}, \varphi_{2}, \ldots, \varphi_{n} \in S$ such that for each $\varphi \in S$ we can find an index $i=1,2, \ldots, n$ for which

$$
\int_{-k}^{-k+1}\left\|\varphi(\theta)-\varphi_{i}(\theta)\right\| d \theta \leqslant \frac{b}{3 a} .
$$


On the other hand, since the space of continuous functions $C([-k,-k+1]$; $X)$ is dense in the space of Bochner - integrable functions ( [4]) we may choose continuous functions $\psi_{1}, \ldots, \psi_{n}$ such that

$$
\int_{-k}^{-k+1}\left\|\varphi_{i}(\theta)-\psi_{i}(\theta)\right\| d \theta<\frac{b}{3 a}, \quad i=1,2, \ldots, n .
$$

We now define the function $q(\theta)=a e^{-\alpha(-k+1-\theta)}$ for $\theta \in[-k,-k+1]$, where $\alpha$ is a positive constant such that

$$
\int_{-k}^{-k+1} q(\theta)\left\|\Psi_{i}(\theta)\right\| d \theta<\frac{b}{3}
$$

for all $i=1,2, \ldots, n$. Therefore, if follows form (2.1), (2.2) and (2.3) that for each $\varphi \in S$ we can choose a function $\varphi_{i}$ for which the following estimate hold

$$
\begin{aligned}
\int_{-k}^{-k+1} q(\theta)\|\varphi(\theta)\| d \theta & <\int_{-k}^{-k+1} q(\theta)\left\|\varphi(\theta)-\varphi_{i}(\theta)\right\| d \theta \\
& +\int_{-k}^{-k+1} q(\theta)\left\|\varphi_{i}(\theta)-\psi_{i}(\theta)\right\| d \theta \\
& +\int_{-k}^{-k+1} q(\theta)\left\|\psi_{i}(\theta)\right\| d \theta \\
& <b
\end{aligned}
$$

which completes the proof.

LEMMA 3. If $S$ is a compact subset of $B$ then there exists a function $g \in G$ such that $S \subseteq B_{g}$ and $S$ is also compact for the topology of $B_{g}$.

Proof. We can assume that $S$ contains a function $\varphi_{0}$ such that $\left\|\varphi_{0}(\theta)\right\|=1$, for $-\infty<\theta<0$. By lemma 2 we may assert that there exists a sequence of conti nuous, positive and increasing functions $q_{k}:[-k,-k+1] \longrightarrow \mathbb{R}, k>1$, such that $q_{1}(0)=1, q_{k}(-k+1)=q_{k-1}(-k+1)$, for $k>2$, and

$$
\int_{-k}^{-k+1} q_{k}(\theta)\|\varphi(\theta) \quad\| d \theta<\frac{1}{2^{k}}
$$

for every $\varphi \in S$ and all $k \in \mathbb{N N}$.

We define the function $h:(-\infty, 0] \longrightarrow$ IR by $h(\theta)=q_{k}(\theta)$ for $-k<\theta<-k+1$, and $k>1$. Then $h$ is a continuous and increasing function such that $h(0)=1$ and

$$
\int_{-\infty}^{0} h(\theta)\|\varphi(\theta)\| d \theta<1,
$$

for every $\varphi \in S$. Substituting $\varphi_{0}$ in the inequality (2.4) we obtain

$$
\int_{-\infty}^{0} h(\theta) d \theta<1
$$


which implies that $h(\theta) \longrightarrow 0$, as $\theta \longrightarrow-\infty$. It follows from this that $h \in G$.

On the other hand, since $S$ is bounded in $B$, there exists a positive constant M such that

$$
\|\varphi(0)\| \leqslant M,
$$

for every $\varphi \in S$.

In view of (2.4) and (2.5) we conclude that $S$ is a set included and bounded in $B_{h}$. Finally, using lemma 1 . ii) we obtain that $S$ is compact in $B_{g}$, where $g=h^{2}$

Our last lemma is the following.

LEMMA 4. Let $S$ be a compact subset of $B$ and let $P: S \longrightarrow B$ be a continuous map. Then there exists a function $g \in G$ such that $S \subseteq B_{g}$ and $P: S \longrightarrow B_{g}$ is continuous for the topology induced by $B_{g}$ in $S$.

Proof. Proceeding now as in the proof of lemma 3 we obtain that there exist $g_{1}, g_{2} \in G$ such that $S \subseteq B_{g_{1}}, P(S) \subseteq B_{g_{2}}$ and the inclusions $i:(S, \tau) \longrightarrow B_{g_{j}}$, are continuous. Let us consider the function $g=g_{1} \cdot g_{2}$. It is clear that $g \in G$ and that the inclusion mappings $B_{g_{i}} \longrightarrow B_{g}$ are continuous. Therefore both $S$ as $\mathrm{P}(\mathrm{S})$ are subsets of $B_{\mathrm{g}}$. Since the map $\mathrm{P}: \mathrm{S} \subseteq B_{\mathrm{g}} \longrightarrow B_{\mathrm{g}}$ can be represented by the composition $S \longrightarrow(\mathrm{P}, \mathrm{P}(\mathrm{S}), \tau) \longrightarrow \mathrm{B}_{\mathrm{g}}$ we obtain that $P$ is continuous for the topology induced by the space $B_{g}$.

We shall now end this note with statement and demonstration of the following.

THEOREM 2. Horn's theorem holds in the space $(B, \tau)$.

Proof. Having obtained the lemmas 1 - 4 we can repeat the proof carried out in [ 1 ] in the context of continuous functions with values in $\mathbb{R}^{n}$. In fact, the proof only depends on the properties established in the lemmas $1-4$. We include it here for completeness.

By lemma 3 there exists $g_{1} \in G$ such that $s_{0}$ and $s_{2}$ arecompact subsets of the space $B_{g_{1}}$. Furthermore, applying lemma 4 we may conclude that there exists $\mathrm{g}_{2} \in G$ such that $\mathrm{P}: \mathrm{S}_{2} \longrightarrow \mathrm{B}_{\mathrm{g}_{2}}$ is continuous for the $B_{\mathrm{g}_{2}}$ - topology. Let us define $g=g_{1} \cdot g_{2}$. Then $S_{o}$ and $S_{2}$ are also compact in $B_{g}$ and $P: S_{2} \longrightarrow B_{g}$ is still continuous. On the other hand, from lemma 1 we obtain that $S_{1}$ is open in $S_{2}$, for the $B_{g}-$ topology in $S_{2}$. Moreover, it is clear that conditions $a$ ) and $b$ ) of theorem 1 remain unchanged. Thus theorem 1 applied in the space $B_{g}$ implies that $P$ has a fixed point in $\mathrm{S}_{\mathrm{o}}$

ACKNOWLEDGEMENT. This work was partially supported by FONDECYT Proyect \#744/89 and DICYT, Proyect 8933HM. 


\section{REFERENCES}

1. T.A. BURTON ; D.P. DWIGGINS, An asymptotic fixed point theorem for a locally convex space, Proc. Amer. Math. Soc., Vol. 103, №1, (1988), 247-251.

2. M.C. DELFOUR, State theory of linear hereditary differential systems, J. Math. Ana1. App1. Vol. 60, Nㅡㄴ 1, (1977), 8-35.

3. W.A. HORN, Some fixed point theorems for compact maps and flows in Banach spaces, Trans. Amer. Math. Soc. 149, (1970), 391-404.

4.- C.M. MARLE, Mesures et Probabilités, Hermann, Paris, 1974. 


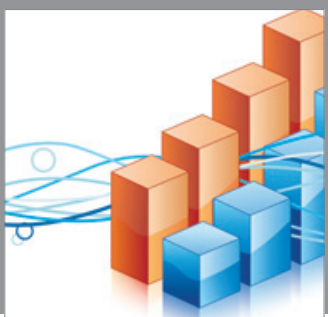

Advances in

Operations Research

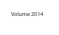

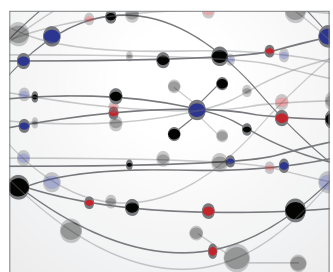

\section{The Scientific} World Journal
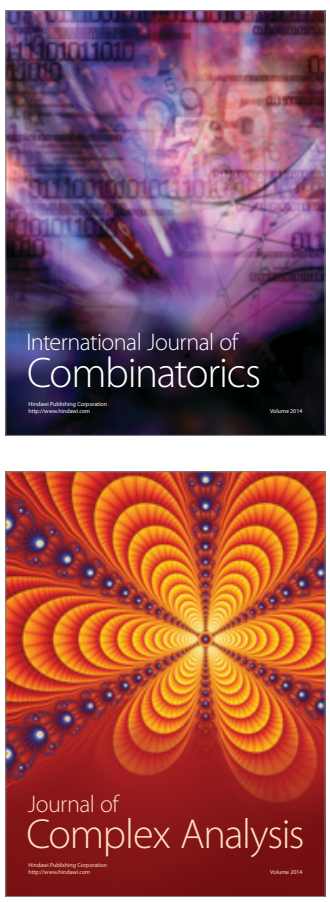

International Journal of

Mathematics and

Mathematical

Sciences
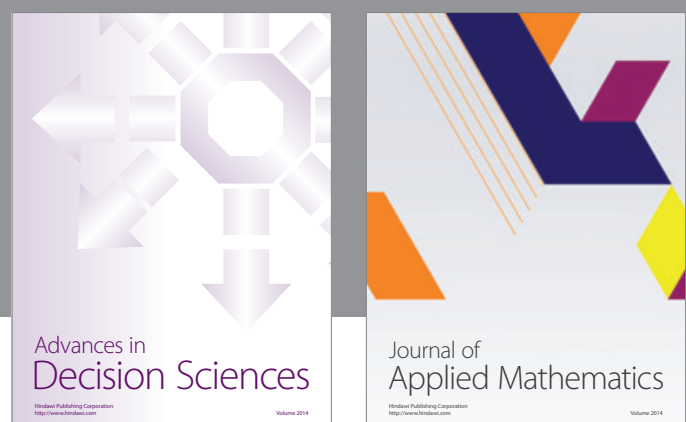

Journal of

Applied Mathematics
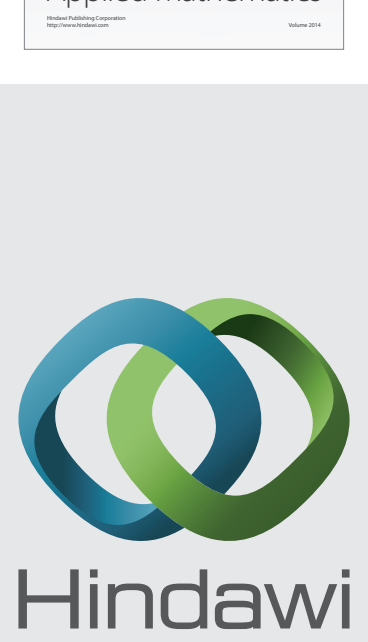

Submit your manuscripts at http://www.hindawi.com
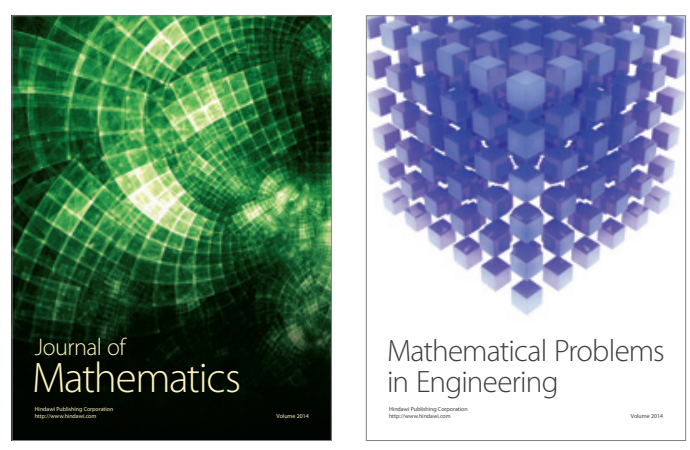

Mathematical Problems in Engineering
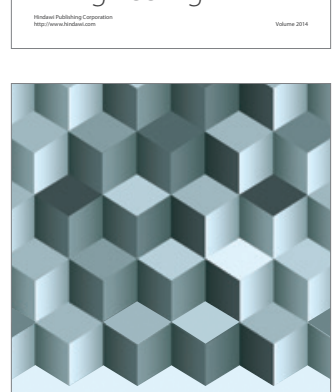

Journal of

Function Spaces
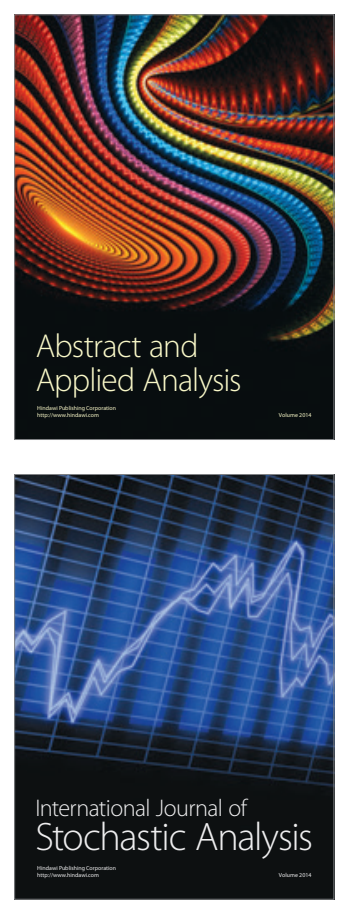

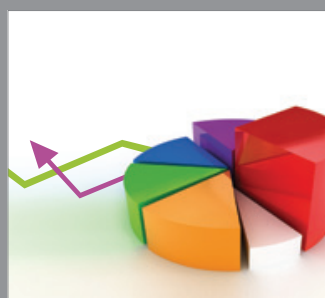

ournal of

Probability and Statistics

Promensencen
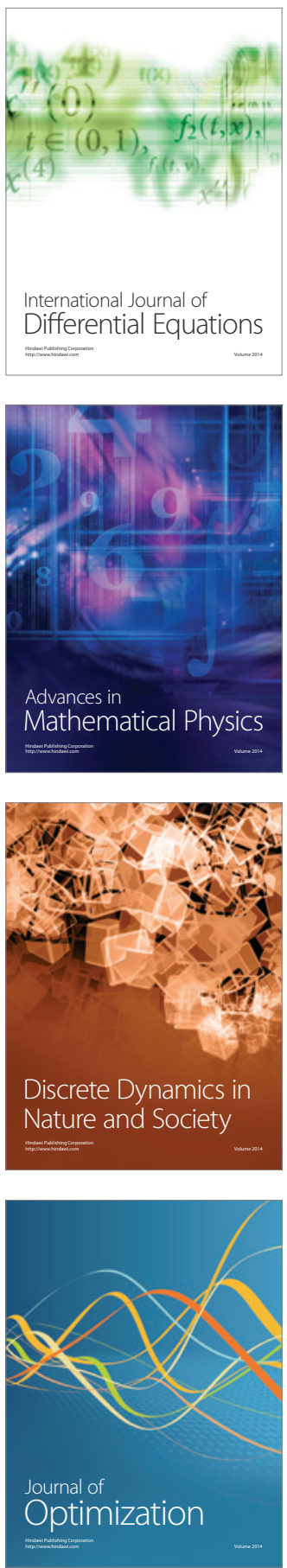